\title{
Substructuring Preconditioners for the Bidomain Extracellular Potential Problem
}

\author{
Micol Pennacchio ${ }^{1}$ and Valeria Simoncini ${ }^{2,1}$ \\ 1 IMATI - CNR, via Ferrata, 1, 27100 Pavia, Italy micol@imati.cnr.it \\ 2 Dipartimento di Matematica, Università di Bologna, Piazza di Porta S. Donato, \\ 5, 40127 Bologna, and CIRSA Ravenna, Italy valeria@dm.unibo.it
}

\begin{abstract}
Summary. We study the efficient solution of the linear system arising from the discretization by the mortar method of mathematical models in electrocardiology. We focus on the bidomain extracellular potential problem and on the class of substructuring preconditioners. We verify that the condition number of the preconditioned matrix only grows polylogarithmically with the number of degrees of freedom as predicted by the theory and validated by numerical tests. Moreover, we discuss the role of the conductivity tensors in building the preconditioner.
\end{abstract}

\section{Introduction}

A macroscopic model accounting for the excitation process in the myocardium is the "bidomain" model that yields the following Reaction-Diffusion (R-D) system of equations for the intra-, extracellular and transmembrane potential $u_{i}, u$ and $v=u_{i}-u$ : find $(v(\boldsymbol{x}, t), u(\boldsymbol{x}, t)), \boldsymbol{x} \in \Omega, t \in[0, T]$ such that

$$
\begin{aligned}
c_{m} \partial_{t} v-\operatorname{div} M_{i} \nabla v+I(v) & =\operatorname{div} M_{i} \nabla u+I_{a p p} & & \text { in } \Omega \times] 0, T[ \\
-\operatorname{div} M \nabla u & =\operatorname{div} M_{i} \nabla v & & \text { in } \Omega \times] 0, T[
\end{aligned}
$$

with $M_{i}, M_{e}, M=M_{i}+M_{e}$ conductivity tensors modeling the cardiac fibers, $I_{a p p}$ an applied current used to initiate the process, $c_{m}$ the surface capacitance of the membrane. The function $I(v)$ is the transmembrane ionic current which is assumed for simplicity to depend only on $v$ and to be a cubic polynomial (see [7]). The general R-D system can be more complex, including additional ordinary differential equations that govern the evolution of $v$.

These models are computationally challenging because of the different space and time scales involved; realistic three-dimensional simulations with uniform grids yield discrete problems with more than $O\left(10^{7}\right)$ unknowns at every time step.

To improve computational efficiency, we consider a non-conforming nonoverlapping domain decomposition, within the mortar finite element method. This allows us to concentrate the computational work only in regions of high 
electrical activity; in addition, the matching of different discretizations on adjacent subdomains are weakly enforced. In $[8,9]$ we compared this technique to the classical conforming FEM verifying its better performance.

In this paper, we focus on the problem of the efficient solution of the linear system arising from this discretization and here for simplicity, we concentrate on the problem with the elliptic equation of (1): for each time instant $t$ find $u(\boldsymbol{x}, t)$, solution of:

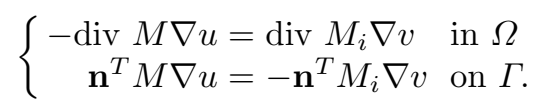

Such problem is of interest in its own right, as it represents a separate model for the bidomain extracellular potential [8].

We consider substructuring preconditioners and we report our numerical experience on solving problem (2). Our experiments confirm the theory depicting polylogarithmic bound for the condition number of the preconditioned matrix. Moreover, attention is devoted to tuning the preconditioner so as to take into account the conductivity tensor $M$ in (2).

\section{Mortar Method}

The computational domain $\Omega$ is decomposed as the union of $L$ subdomains $\Omega_{1}, \ldots, \Omega_{L}$ (see, e.g., [10]). We set $\Gamma_{\ell n}=\partial \Omega_{n} \cap \partial \Omega_{\ell}, \mathcal{S}=\cup \Gamma_{\ell n}$ and we denote by $\gamma_{\ell}^{(i)}, i=1, \ldots, 4$ the $i$-th side of the $\ell$-th domain so that $\partial \Omega_{\ell}=\bigcup_{i=1}^{4} \gamma_{\ell}^{(i)}$. Here we consider only geometrically conforming decomposition, i.e. each edge $\gamma_{l}^{(i)}$ coincides with $\Gamma_{l n}$ for some $n$.

The Mortar Method is applied by choosing a splitting of the skeleton $\mathcal{S}$ as the disjoint union of a certain number of subdomain sides $\gamma_{l}^{(i)}$, called mortar or slave sides: we fix an index set $I \subset\{1, \ldots, L\} \times\{1, \ldots, 4\}$ such that $\mathcal{S}=\bigcup_{(l, i) \in I} \gamma_{l}^{(i)}$. The index-set corresponding to trace or master sides will be denoted by $I^{*}: I^{*} \subset\{1, \ldots, L\} \times\{1, \ldots, 4\}, I^{*} \cap I=\emptyset$ and $S=\bigcup_{(l, i) \in I^{*}} \gamma_{l}^{(i)}$.

Let the spaces $X$ and $T$ be $X=\prod_{\ell} H^{1}\left(\Omega_{\ell}\right), T=\prod_{\ell} H^{1 / 2}\left(\partial \Omega_{\ell}\right)$ with the broken norms: $\|u\|_{X}^{2}=\sum_{\ell}\|u\|_{1, \Omega_{\ell}}^{2}$ and $\|\eta\|_{T}^{2}=\sum_{\ell}\left\|\eta_{\ell}\right\|_{1 / 2, \partial \Omega_{\ell}}^{2}$. For each $\ell$, let also $\mathcal{V}_{h}^{\ell}$ be a family of finite dimensional subspaces of $H^{1}\left(\Omega_{\ell}\right) \cap C^{0}\left(\bar{\Omega}_{\ell}\right)$, depending on a parameter $h=h_{\ell}>0, X_{h}=\prod_{\ell=1}^{L} \mathcal{V}_{h}^{\ell} \subset X, T_{h}^{\ell}=\left.\mathcal{V}_{h}^{\ell}\right|_{\partial \Omega_{\ell}}$ and $T_{h}=\prod_{\ell=1}^{L} T_{h}^{\ell} \subset T$. Then we define two composite bilinear forms $a_{X}, a_{X}^{i}$ : $X \times X \longrightarrow \mathbb{R}$ as:

$$
a_{X}(u, \phi)=\sum_{\ell} \int_{\Omega_{\ell}} \nabla \phi_{l}^{T} M \nabla u_{\ell} d x, \quad a_{X}^{i}(u, \phi)=\sum_{\ell} \int_{\Omega_{\ell}} \nabla \phi_{l}^{T} M_{i} \nabla u_{\ell} d x .
$$

Since these bilinear forms are not coercive on $X$, we consider proper subspaces of $X$ consisting of functions satisfying a suitable weak continuity constraint, leading to the following constrained approximation and trace spaces 


$$
\begin{gathered}
\mathcal{X}_{h}=\left\{v_{h} \in X_{h}, \int_{S}\left[v_{h}\right] \lambda d s=0, \forall \lambda \in M_{h}\right\} \\
\mathcal{T}_{h}=\left\{\eta \in T_{h}, \int_{S}[\eta] \lambda d s=0, \forall \lambda \in M_{h}\right\},
\end{gathered}
$$

with $M_{h}$ a suitably chosen finite dimensional multiplier space. We can write the discrete problem, whose solution existence was proved in [8, Theorem 3.1]:

Problem 1. Find $u_{h} \in \mathcal{X}_{h}$ such that for all $\phi_{h} \in \mathcal{X}_{h}$

$$
a_{X}\left(u_{h}, \phi_{h}\right)=-a_{X}^{i}\left(v_{h}, \phi_{h}\right) .
$$

We remark that Problem 1 admits a solution unique up to an additive constant related to the reference potential chosen. In this paper we consider as reference potential the one given by the potential at a reference point $\boldsymbol{x}_{0} \in \Omega$.

\section{Substructuring Preconditioners}

A key aspect of substructuring preconditioners is that they distinguish among three types of degrees of freedom: interior (corresponding to basis functions vanishing on the skeleton and supported on one sub-domain), edge and vertex degrees of freedom $[4,1]$. Thus, each function $u \in \mathcal{X}_{h}$ can be written as the sum of three suitably defined components: $u=u^{0}+u^{E}+u^{V}$. More specifically, let $w=\left(w_{\ell}\right)_{\ell=1, \cdots, L} \in X_{h}$ be any discrete function, then

$$
w=w^{0}+R_{h}(w), \quad w^{0} \in \mathcal{X}_{h}^{0}
$$

with $w^{0} \in \mathcal{X}_{h}^{0}$ interior function and $R_{h}(w)$ a discrete lifting, i.e. $R_{h}(w)=$ $\left(R_{h}^{\ell}\left(w_{\ell}\right)\right)_{\ell=1, \ldots, K}$, where $R_{h}^{\ell}\left(w_{\ell}\right)$ is the unique element in $\mathcal{V}_{h}^{\ell}$ satisfying $R_{h}^{\ell}\left(w_{\ell}\right)$ $=w_{\ell}$ on $\Gamma_{\ell}$ and

$$
\int_{\Omega_{\ell}} \sum_{i, j} M \frac{\partial}{\partial \boldsymbol{x}_{i}} \frac{\partial}{\partial \boldsymbol{x}_{j}} R_{h}^{\ell}\left(w_{\ell}\right) v_{h}^{\ell} d x=0, \quad \forall v_{h} \in \mathcal{V}_{h}^{\ell}
$$

Consequently, the spaces $X_{h}$ and $\mathcal{X}_{h}$ can be split as:

$$
X_{h}=X_{h}^{0} \oplus R_{h}\left(T_{h}\right) \quad \mathcal{X}_{h}=\mathcal{X}_{h}^{0} \oplus R_{h}\left(\mathcal{T}_{h}\right)
$$

and it can be verified that

$$
a_{X}(w, v)=a_{X}\left(w^{0}, v^{0}\right)+a_{X}\left(R_{h}(w), R_{h}(v)\right)=a_{X}\left(w^{0}, v^{0}\right)+s(\eta(w), \eta(v)),
$$

where the discrete Steklov-Poincaré operator $s: T_{h} \times T_{h} \rightarrow \mathbb{R}$ is defined by

$$
s(\xi, \eta):=\sum_{\ell} \int_{\Omega_{\ell}}\left(M(\boldsymbol{x}) \nabla R_{h}^{\ell}(\xi)\right) \cdot \nabla R_{h}^{\ell}(\eta) .
$$


Furthermore the space of constrained skeleton functions $\mathcal{T}_{h}$ can be split as the sum of vertex and edge functions. More specifically, denoting by $\mathfrak{L} \subset$ $\prod_{\ell=1}^{L} H^{1 / 2}\left(\partial \Omega_{\ell}\right)$ the space $\mathfrak{L}=\left\{\left(\eta_{\ell}\right)_{\ell=1, \cdots, L}, \eta_{\ell}\right.$ is linear on each edge of $\left.\Omega_{\ell}\right\}$, then we can define the space of constrained vertex functions as

$$
\mathcal{T}_{h}^{V}=\mathcal{P}_{h} \mathfrak{L}
$$

with $\mathcal{P}_{h}$ the correction operator imposing the constraint. We make the (not restrictive) assumption $\mathfrak{L} \subset T_{h}$, which yields $\mathcal{T}_{h}^{V} \subset \mathcal{T}_{h}$, and we introduce the space of constrained edge functions $\mathcal{T}_{h}^{E} \subset \mathcal{T}_{h}$ defined by

$$
\mathcal{T}_{h}^{E}=\left\{\eta=\left(\eta_{\ell}\right)_{\ell=1, \cdots, L} \in \mathcal{T}_{h}, \eta_{\ell}(A)=0, \forall \operatorname{vertex} A \text { of } \Omega_{\ell}\right\} .
$$

We can easily verify that $\mathcal{T}_{h}=\mathcal{T}_{h}^{V} \oplus \mathcal{T}_{h}^{E}$.

Then we will consider a block Jacobi type preconditioner $\tilde{s}: \mathcal{T}_{h} \times \mathcal{T}_{h} \longrightarrow \mathbb{R}$ defined as

$$
\tilde{s}(\eta, \xi)=b^{V}\left(\eta^{V}, \xi^{V}\right)+b^{E}\left(\eta^{E}, \xi^{E}\right)
$$

with blocks related to the following edge and vertex global bilinear forms

$$
\begin{array}{lll}
b^{E}: \mathcal{T}_{h}^{E} \times \mathcal{T}_{h}^{E} \longrightarrow \mathbb{R} & \text { such that } & b^{E}\left(\eta^{E}, \eta^{E}\right) \simeq s\left(\eta^{E}, \eta^{E}\right) \\
b^{V}: \mathcal{T}_{h}^{V} \times \mathcal{T}_{h}^{V} \longrightarrow \mathbb{R} & \text { such that } & b^{V}\left(\eta^{V}, \eta^{V}\right) \simeq s\left(\eta^{V}, \eta^{V}\right)
\end{array}
$$

\subsection{Matrix form}

In this section we derive the matrix form of the discrete Steklov-Poincaré operator $s$ in (10). Equation (6) yields the following linear system of equations:

$$
A \mathbf{u}=\mathbf{b} \quad \text { with } \quad \mathbf{b}=-A_{i} \mathbf{v},
$$

where $A, A_{i}$ are the stiffness matrices associated to the discretization of $a_{X}, a_{X}^{i}$ defined in (3). It can be shown that the matrix $A$ is positive semidefinite and the system is consistent.

We reorder the vector of unknowns as: $\mathbf{u}=\left(\mathbf{u}_{0}, \mathbf{u}_{E}, \mathbf{u}_{V}, \mathbf{u}_{S}\right)^{T}$, with $\mathbf{u}_{0}, \mathbf{u}_{E}, \mathbf{u}_{V}, \mathbf{u}_{S}$ interior, edge, vertex and slave nodes, respectively. From the mortar condition, it follows that the interior nodes of the multiplier sides are not associated with genuine degrees of freedom in the FEM space. Indeed, the value of the coefficients $\mathbf{u}_{S}$ corresponding to basis functions "living" on slave sides is uniquely determined by the remaining coefficients through the jump (mortar) condition and can be eliminated from the global vector $\mathbf{u}$, i.e.

$$
C_{S} \mathbf{u}_{S}=-C_{E} \mathbf{u}_{E}-C_{V} \mathbf{u}_{V} \quad \mathbf{u}_{S}=: Q_{E} \mathbf{u}_{E}+Q_{V} \mathbf{u}_{V}
$$

where $Q_{E}=-C_{S}^{-1} C_{E}, Q_{V}=-C_{S}^{-1} C_{V}$. The entries of $C_{S}, C_{E}, C_{V}$ are given by $c_{i j}=\int_{\gamma_{m}}\left[\phi_{j}\right] \lambda_{i} d s, \lambda_{i} \in M_{h}$ with $\phi_{j}$ corresponding to the different nodal basis functions on the slave and master side and associated with the vertices. Since biorthogonal basis functions are employed, the square matrix $C_{S}$ is 
diagonal and easily invertible (cf. [11]). The reduction in (16) may be written in matrix form as

$$
\mathbf{u}=Q\left(\begin{array}{l}
\mathbf{u}_{0} \\
\mathbf{u}_{E} \\
\mathbf{u}_{V}
\end{array}\right) \quad \text { with } \quad Q=\left(\begin{array}{ccc}
I_{0} & 0 & 0 \\
0 & I_{E} & 0 \\
0 & 0 & I_{V} \\
0 & Q_{E} & Q_{V}
\end{array}\right)
$$

where $Q$ is a global "switching" matrix. The resulting reduced system is thus given by

$$
\widetilde{\mathcal{A}} \mathbf{u}_{M}=\tilde{\mathbf{b}}
$$

with $\widetilde{\mathcal{A}}=Q^{T} \mathcal{A} Q$ and $\tilde{\mathbf{b}}=Q^{T} \mathbf{b}$. We note that the $(1,1)$ block in $\widetilde{\mathcal{A}}$ is cheaply invertible therefore, the Schur complement of the system relative to the $(1,1)$ block can readily be obtained, yielding the further reduced system

$$
S\left(\begin{array}{c}
\mathbf{u}_{E} \\
\mathbf{u}_{V}
\end{array}\right)=\left(\begin{array}{c}
\widehat{\mathbf{b}}_{E} \\
\widehat{\mathbf{b}}_{V}
\end{array}\right) .
$$

The Schur complement $S$ represents the matrix form of the Steklov-Poincaré operator $s(\cdot, \cdot)$. To obtain the matrix form of $\tilde{s}(\cdot, \cdot)$ we consider the space $\mathfrak{L}$ of linear functions, used in the splitting of the trace space (11). Then, we introduce an interpolation map denoted by $R_{H}^{T}$ (say piecewise interpolation) from the nodal value on $V$ (vertices) onto all nodes of $\mathcal{S}$. The matrix $R_{H}$ can be viewed as the weighted restriction map from $\mathcal{S}$ onto $V$. By defining the square matrix $J=\left(\left(\begin{array}{c}I_{E} \\ O\end{array}\right) R_{H}\right)$, with $I_{E}$ the $n_{E} \times n_{E}$ identity matrix, we can derive the new Schur complement matrix $\widetilde{S}$, after the "vertex" correction,

$$
\widetilde{S}=J^{T} S J=\left(\begin{array}{cc}
\widetilde{S}_{E} & \widetilde{S}_{E V} \\
\widetilde{S}_{E V}^{T} & \widetilde{S}_{V}
\end{array}\right)
$$

\subsection{The preconditioner}

We describe a generalization of a known optimal preconditioner for $\widetilde{S}$, and some more computationally effective variants. The matrix discretization of the form $\tilde{s}$ yields the following (block-Jacobi type) diagonal preconditioner

$$
P=\left(\begin{array}{cc}
P_{E} & 0 \\
0 & P_{V}
\end{array}\right)
$$

where $P_{E}, P_{V}$ are the matrix counterparts of the bilinear forms $b^{E}$ and $b^{V}$ in (14), respectively.

It can be verified that the preconditioned matrix $P^{-1} S$ satisfies the theory developed in $[1,3]$ so that

$$
\operatorname{cond}\left(P^{-1} \widetilde{S}\right) \lesssim\left(1+\log \left(\frac{H}{h}\right)\right)^{2}
$$


with $H$ size of the subdomains and $h$ finest meshsize of the finite element spaces used. Moreover, if an auxiliary coarse mesh is chosen for the vertex block with mesh size $\delta>h$ as studied in [3], then a similar estimate can be obtained but with a factor $\left(1+\log \left(\frac{H}{h}\right)\right)^{3}$.

The next three variants make the preconditioner above computationally more appealing with no essential loss of optimality. This goal is achieved by replacing either or both the edge and vertex blocks $P_{E}$ and $P_{V}$ with more convenient approximations. In their construction, we were inspired by a similar approach first proposed in $[4,1,3]$ for elliptic problems. For later considerations, we recall here an important bound for the condition number of the preconditioned matrix, expressed in terms of the preconditioning quality of the two diagonal blocks. More precisely, let $P=\operatorname{diag}\left(P_{1}, P_{2}\right)$ be a Jacobitype preconditioner, and let $\mu_{M}=\max \left\{\lambda_{\max }\left(P_{1}^{-1} \tilde{S}_{E}\right), \lambda_{\max }\left(P_{2}^{-1} \tilde{S}_{V}\right)\right\}, \mu_{m}=$ $\min \left\{\lambda_{\min }\left(P_{1}^{-1} \tilde{S}_{E}\right), \lambda_{\min }\left(P_{2}^{-1} \tilde{S}_{V}\right)\right\}$. Then

$$
\operatorname{cond}\left(P^{-1} \tilde{S}\right) \leq \frac{1+\gamma}{1-\gamma} \frac{\mu_{M}}{\mu_{m}} \quad \gamma \leq 1
$$

where $1+\gamma$ is the largest eigenvalue of the preconditioned matrix obtained by using the block diagonal of $\tilde{S}$ as preconditioner $P$ (see, e.g., [6]).

Following $[1,4]$ a simple approach consists in dropping all couplings between different edges and between edges and vertex points: $P_{E}$ is replaced by its block diagonal part with one block for each mortar. This simplification provides our first variant,

$$
P_{1}=\left(\begin{array}{cc}
P_{E}^{\text {diag }} & 0 \\
0 & P_{V}
\end{array}\right)
$$

Assembling the edge and vertex block preconditioner with such a choice could be quite expensive. A more efficient preconditioner may be obtained by approximating the edge block $P_{E}$ of $P$ as

$$
P_{E}^{(R)}=\alpha R
$$

where $R$ is the square root of the stiffness matrix associated on each edge to the discretization of the operator $-d^{2} / d x^{2}$ with homogeneous Dirichlet conditions at the extrema $[4,5,10]$. The choice of the parameter $\alpha$ is discussed below. Thus our second variant is

$$
P_{2}=\left(\begin{array}{cc}
P_{E}^{(R)} & 0 \\
0 & P_{V}
\end{array}\right)
$$

It can be easily verified that (cf., e.g., $[4,5]$ )

$$
c_{1} \mathbf{v}^{T} \widetilde{S}_{E} \mathbf{v} \leq \mathbf{v}^{T} R \mathbf{v} \leq c_{2}\left(1+\log \left(\frac{H}{h}\right)\right)^{2} \mathbf{v}^{T} \widetilde{S}_{E} \mathbf{v}
$$


where $c_{1}, c_{2}$ are independent of $H, h$ but may depend on the coefficients of $M(\boldsymbol{x})$. Since $P_{V}=\widetilde{S}_{V}$, in (21) we obtain $\mu_{M}=\max \left\{\lambda_{\max }\left((\alpha R)^{-1} \tilde{S}_{E}\right), 1\right\} \leq$ $\max \left\{\alpha^{-1} c_{2}\left(1+\log \left(\frac{H}{h}\right)\right)^{2}, 1\right\}$. Analogously, $\mu_{m} \geq \min \left\{\alpha^{-1} c_{1}, 1\right\}$. Therefore, the determination of $\mu_{M}, \mu_{m}$ is influenced by the magnitude of $c_{1}, c_{2}$ and of $\alpha$. The anisotropic conductivity tensor $M=M_{i}+M_{e}$ is given as $M_{s}=M_{s}(\boldsymbol{x})=$ $\sigma_{t}^{s} I+\left(\sigma_{l}^{s}-\sigma_{t}^{s}\right) \mathbf{a a}^{T}, s=i, e$, where $\mathbf{a}=\mathbf{a}(\boldsymbol{x})$ is the unit vector tangent to the cardiac fiber at a point $\boldsymbol{x} \in \Omega, I$ is the identity matrix and $\sigma_{l}^{s}, \sigma_{t}^{s}$ for $s=i, e$ are the conductivity coefficients along and across fiber, in the (i) and (e) media, assumed constant with $\sigma_{l}^{s}>\sigma_{t}^{s}>0$. As already mentioned, the magnitude of $c_{1}, c_{2}$ depends on the conductivity coefficients. Therefore, to minimize the bound on the condition number in (21), it is standard practice to select $\alpha$ of the same order of magnitude as the conductivity coefficients [5]. In our case, by choosing $\alpha$ as $\|M\| \leq 2 \sigma_{t}+\sigma_{\ell}=$ : $\alpha$ we optimize the upper bound $\mu_{M}$ with respect to the conductivity coefficients. Since $\alpha \ll 1$, this value of $\alpha$ usually also leads to the lower estimate $\mu_{m}=1$. Numerical experiments validated this choice.

Our third variant copes with the already mentioned fact that building $P_{V}$ becomes expensive when grid refinements are required. Various choices have been discussed in the literature [10]; for instance, in [3] the vertex preconditioner was chosen as the vertex block of the Schur complement matrix on a fixed auxiliary coarse mesh, independent of the space discretization. We thus approximate $P_{V}$ with the matrix $P_{V c}$ obtained with a fixed coarse mesh, yielding

$$
P_{3}=\left(\begin{array}{cc}
P_{E}^{(R)} & 0 \\
0 & P_{V c}
\end{array}\right)
$$

This variant leads to very moderate (close to unit) values of $\lambda_{\min }\left(P_{V c}^{-1} \widetilde{S}_{V}\right)$ and $\lambda_{\max }\left(P_{V c}^{-1} \widetilde{S}_{V}\right)$ to achieve an estimate in (21). Therefore, we maintained the selection of $\alpha$ as discussed above.

In Table 1 we report numerical experiments with the preconditioners $P_{1}, P_{2}$ and $P_{3}$ for the Schur complement system associated with the matrix in (19). The results are in close agreement with the theory: the condition number of the preconditioned matrix grows at most polylogarithmically with the number of degrees of freedom per subdomain, as indicated by (20). The columns with $\alpha=1$ refer to such parameter selection in $P_{E}^{(R)}$. This corresponds to discarding information on the conductivity tensor $M$ in (2). The worse convergence validates our choice and shows the importance of an appropriate choice of the parameter. Note that the selected value of $\alpha$ was more effective than other choices of similar magnitude. Indeed choosing $\alpha=\sigma_{l}$ and $\alpha=\sigma_{t}$ for $N^{2}=256$, $n=5,10,20,40$ (fourth row in the table) and $P_{3}$, we obtained 29,31,33,34 and $42,45,48,49$, respectively. In summary, our experiments demonstrate that the proposed variants allow us to limit the computational cost (the cost of forming $P_{E}^{(R)}$ and $P_{V_{c}}$ is much lower than that for their original counterparts), with basically no loss in convergence rate, for the appropriate scaling factor. 
Table 1. Number of conjugate gradient iterations needed to reduce the residual of a factor $10^{-5}$ with the preconditioners $P_{1}, P_{2}, P_{3}$ and $P_{2}, P_{3}$ with $\alpha=1 . K=N^{2}$ : \# of subdomains. $n^{2}$ : \# of elements per subdomain. Symbol '*': the preconditioner $P_{1}$ could not be built due to memory constraints.

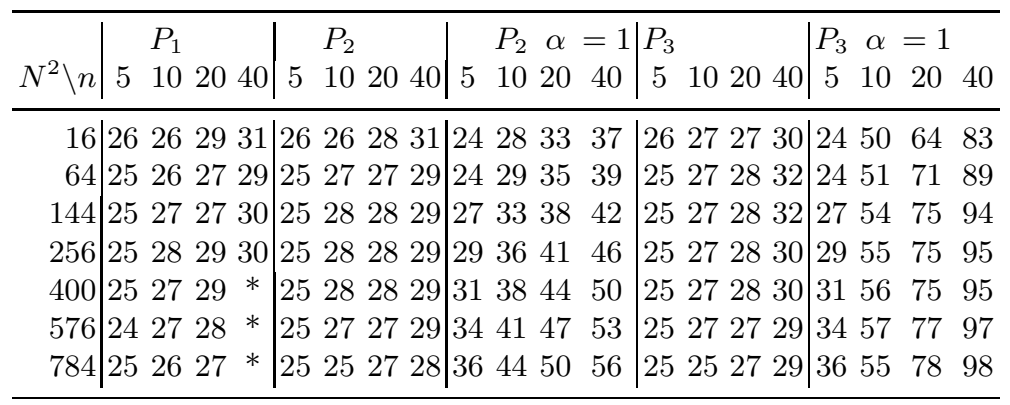

\section{References}

1. Achdou, Y., Maday, Y., Widlund, O.: Substructuring preconditioners for the mortar method in dimension two. SIAM J. Numer. Anal., 36, 551-580 (1999)

2. Bernardi, C., Maday, Y., Patera, A.T: Domain decomposition by the mortar element method. In: Kaper, H. et al. (eds) Asymptotic and Numerical Method for Partial Differential Equations with Critical Parameters. Dordrecht: Reidel, (1993)

3. Bertoluzza, S., Pennacchio, M.: Preconditioning the mortar method by substructuring: the high order case. Appl. Num. Anal. Comp. Math., 1, 434-454 (2004)

4. Bramble, J. H., Pasciak, J.E., Schatz, A. H.: The construction of preconditioners for elliptic problems by substructuring, I. Math. Comp., 47, 103-134 (1986)

5. Chan, T. F., and Mathew, T. P., Domain Decomposition Algorithms, In: Acta Numerica 1994, Cambridge University Press, 61-143 (1994)

6. Y. Notay, Algebraic multigrid and algebraic multilevel methods: a theoretical comparison. Numer. Lin. Alg. Appl., 12, 419-451 (2005)

7. Pennacchio, M. and Simoncini, V.: Efficient algebraic solution of reactiondiffusion systems for the cardiac excitation process. J. Comput. Appl. Math., 145 (1): 49-70 (2002)

8. Pennacchio, M. : The mortar finite element method for the cardiac "bidomain" model of extracellular potential, J. Sci. Comput., 20, n.2, pag. 191-210 (2004)

9. Pennacchio, M.: The Mortar Finite Element Method for Cardiac ReactionDiffusion Models, Computers in Cardiology 2004; IEEE Proc., 31: 509-512 (2004)

10. Toselli, A., Widlund, O. Domain decomposition methods - algorithms and theory, Springer Series in Computational Mathematics, volume 34 Springer-Verlag, Berlin (2005)

11. Wohlmuth, B.: Discretization Methods and Iterative Solvers Based on Domain Decomposition, Lecture Notes in Computational Science and Engineering, volume 17, Springer (2001). 